Service social

\title{
Empowerment et service social : approches et enjeux
}

\section{William A. Ninacs}

Volume 44, numéro 1, 1995

Valeurs, pratiques, action sociale

URI : https://id.erudit.org/iderudit/706681ar

DOI : https://doi.org/10.7202/706681ar

Aller au sommaire du numéro

Éditeur(s)

École de service social de l'Université Laval

ISSN

1708-1734 (numérique)

Découvrir la revue

Citer cet article

Ninacs, W. A. (1995). Empowerment et service social : approches et enjeux. Service social, 44(1), 69-93. https://doi.org/10.7202/706681ar

\section{Résumé de l'article}

Il existe diverses approches dites d'empowerment pour chacune des méthodes du service social. Une étude de ces approches permet de conclure que l'empowerment s'avère un processus d'appropriation à dimensions multiples qui s'opère simultanément sur au moins quatre plans : la participation, l'acquisition de connaissances spécifiques, l'estime de soi et la conscience critique. L'interaction de ces composantes caractériserait le processus d'empowerment, car chacune semble y jouer un rôle précis. Cependant, un état d'empowerment est également la finalité du processus du même nom. Un regard sur les liens entre ces différentes dimensions peut aider à cerner certains enjeux de l'intervention axée sur ce phénomène. 


\section{Empowerment et service social: approches et enjeux ${ }^{*}$}

William A. NINACS*

Étudiant au doctorat

École de service social, Université Laval Enseignant et consultant en développement

\section{INTRODUCTION}

De façon générale, il existe une longue tradition d'empowerment en service social même si l'apparition du mot est relativement récente. Dans son étude de l'histoire du service social aux États-Unis, Barbara Levy Simon discerne la présence d'un volet d'empowerment dans I'intervention sociale américaine depuis les settlement houses du siècle dernier (1994: 16). La situation ne serait guère différente dans d'autres pays, car l'empowerment se retrouve à la fois comme approche, processus et finalité ${ }^{1}$ partout où des intervenants et intervenantes tentent d'appuyer les efforts autonomes des individus et des collectivités pour se tailler une place à part égale et à part entière sur l'échiquier social.

* Une version préliminaire de ce texte a été présentée au Colloque de la section Travail social, Association canadienne-française pour l'avancement des sciences (ACFAS), 25 mai 1995, Chicoutimi.

** L'auteur est enseignant au programme de maîtrise en développement économique communautaire au New Hampshire College, Manchester, New Hampshire. 
Cependant, une confusion règne en service social autour du concept d'empowerment (Breton, 1994 : 28) et la conceptualisation de l'empowerment ne concorde pas toujours entre praticiens et auteurs (Davis, 1991 : 58). Cette ambiguïté généralisée est un obstacle sérieux à son utilisation. C'est un peu dommage, car l'empowerment constitue un outil pertinent pour le service social. Ce texte a pour objectif de réduire un peu l'équivoque en relevant les approches utilisées en service social où l'empowerment est spécifiquement présent et en présentant, par la suite, une conceptualisation du processus d'empowerment dans le but de cerner certains enjeux pour le service social.

\section{APPROCHES AXÉES SUR L'EMPOWERMENT EN SERVICE SOCIAL}

Toute discussion sur l'empowerment part du constat que certaines collectivités et certaines personnes ne possèdent pas, ou ont la perception de ne pas posséder, le pouvoir sur les ressources dont elles ont besoin pour assurer leur bien-être. Toute approche axée sur l'empowerment est fondée sur la croyance que les personnes, tant individuellement que collectivement, ont ou peuvent acquérir les capacités pour effectuer les transformations nécessaires pour assurer leur accès à ces ressources, voire même les contrôler.

L'idée que les êtres humains soient capables d'agir pour assurer leur bien-être est intrinsèque au service social (Grosser et Mondros, 1985; Laforest, 1984: 28; Rothman, 1995 : 22). Une approche axée sur l'empowerment cherche ainsi en service social à soutenir les individus et les collectivités dans leurs démarches pour se procurer le pouvoir dont elles ont besoin. La pratique qui en découle comprend cinq éléments essentiels: une collaboration partenariale avec les clients et clientes et avec les mandants [constituents]; une intervention misant sur leurs capacités, leurs forces et leurs ressources; une cible de travail double, c'est-à-dire à la fois sur l'individu et sur son environnement; un postulat opérationnel qui conçoit les clients et clientes comme sujets actifs et comme des ayants droit plutôt que comme des bénéficiaires; une orientation consciente des énergies professionnelles vers des individus et des groupes historiquement ou actuellement opprimés (Mullender et Ward, 1991:23; Simon, 1994: 8-24). L'approche d'empowerment tranche ainsi avec les philosophies axées sur la bienfaisance et la charité, car elle entrevoit le rapport entre tous les individus, y compris celui entre intervenants et usagers, comme étant fondé sur la réciprocité et étant foncièrement 
égalitaire (Weick, 1981 : 180-182). D'une certaine façon, I'empowerment, c'est l'antithèse du paternalisme (Staples, 1990: 30).

Un examen d'ouvrages en service social traitant de l'empowerment permet de constater que les éléments identifiés par Simon peuvent se retrouver, explicitement ou implicitement, à l'intérieur de plusieurs approches utilisées dans les méthodes du service social, bien que ces approches ne lui soient pas exclusives.

\section{Tableau 1}

\section{Approches axées sur l'empowerment en service social}

\begin{tabular}{|c|c|c|}
\hline Méthode & Approche & Quelques auteurs \\
\hline traitement individuel & $\begin{array}{l}\text { - psychologie } \\
\text { communautaire } \\
\text { - structurelle }\end{array}$ & $\begin{array}{l}\text { - Le Bossé et Lavallée (1993) } \\
\text { - Moreau (1989); } \\
\text { Moreau et al. (1993) }\end{array}$ \\
\hline service social des groupes & $\begin{array}{l}\text { - groupe d'entraide } \\
\text { - groupe autogéré }\end{array}$ & $\begin{array}{l}\text { - Lee }(1991 ; 1994) \\
\text { - Mullender et Ward (1991); } \\
\text { Lindsay }(1992)\end{array}$ \\
\hline $\begin{array}{l}\text { organisation communautaire } \\
\text { - action sociale } \\
\text { - planning social }\end{array}$ & $\begin{array}{l}\text { - } \text { pour l'ensemble } \\
\text { - grassroots model } \\
\text { - action communautaire } \\
\text { de défense des droits } \\
\text { sociaux }\end{array}$ & $\begin{array}{l}\text { - Rothman (1995) } \\
\text { - Mondros et Wilson (1994) } \\
\text { - Mayer et Panet-Raymond } \\
\text { (1991) }\end{array}$ \\
\hline $\begin{array}{l}\text { - développement local } \\
\text { de type communautaire }\end{array}$ & $\begin{array}{l}\text { - publique et parapublique } \\
\text { - communautaire } \\
\text { - toutes les stratégies } \\
\text { de cette méthode }\end{array}$ & $\begin{array}{l}\text { - Hardina et Malott (1993) } \\
\text { - Grosser et Mondros (1985) } \\
\text { - Rothman (1987); } \\
\text { Doucet et Favreau (1991); } \\
\text { Miller (1991) }\end{array}$ \\
\hline $\begin{array}{l}\text { administration } \\
\text { de programmes }\end{array}$ & $\begin{array}{l}\text { - empowerment evaluation } \\
\text { - évaluation participative }\end{array}$ & $\begin{array}{l}\text { - Fetterman (1994) } \\
\text { - Papineau et Kiely (1996) }\end{array}$ \\
\hline recherche & - recherche participative & $\begin{array}{l}\text { - Whitmore et Kerans } \\
\text { (1988); Whitmore (1991) }\end{array}$ \\
\hline
\end{tabular}

\section{Le traitement individuel}

Dans une approche $\mathrm{d}^{\prime}$ empowerment, le traitement individuel renvoie davantage à la psychologie communautaire qu'aux autres approches psychologiques ou psychosociales. L'intervention aura pour but $\mathrm{d}^{\prime}$ aider la personne à développer, "par l'intermédiaire d'actions concrètes, le sentiment qu'il lui est possible d'exercer un plus grand contrôle sur les aspects de sa réalité psychologique et sociale qui sont importants pour elles» (Le Bossé et Lavallée, 1993: 17). Il s'agit «d'une réappropriation active du pouvoir thérapeutique » par l'individu et de la mise en route d'un processus continu de contrôle sur 
une ou plusieurs sphères de sa vie (ibid.: 13-17). La modification de la relation de pouvoir de l'individu avec l'intervenant ou l'intervenante et la modification de la relation de pouvoir de l'individu avec son environnement sont donc simultanément visées.

$C^{\prime}$ est dans I'approche structurelle que l'empowerment se retrouve de façon explicite comme fondement de l'intervention de traitement individuel. L'approche structurelle est fondée sur le postulat que, typiquement, les pratiques du service social maintiennent le pouvoir de l'intervenant au détriment de celui du client ou de la cliente. Ces pratiques peuvent toutefois être modifiées par le partage $d^{\prime}$ informations avec le client ou la cliente et par l'implication de celui-ci ou celle-ci dans les décisions concernant l'intervention à son égard. L'effet de l'approche structurelle sera donc d'accroître le pouvoir du client dans sa relation avec l'intervenant et d'augmenter la capacité du client d'être actif dans la relation d' "aide" (Moreau, 1989: 79-82).

\section{Le service social des groupes}

En matière de service social des groupes, il existe différents cadres conceptuels. À titre d'exemple, Lee propose un travail d'empowerment à partir du groupe d'entraide dans lequel I'objectif sera: a) d'amener chaque membre du groupe à se percevoir comme agent de changement [causal agent] et l'intervenant comme partenaire; b) d'aider les individus à percevoir la structure de pouvoir comme multipolaire et donc influençable (1991: 12). Les méthodes sont teintées des mêmes couleurs que l'approche structurelle: relation d'aide axée sur la collaboration, la confiance et le partage du pouvoir; définition du problème par le client ou la cliente; identification des forces du client pour s'en servir comme fondements de l'intervention; implication active du client dans le processus de changement...

Le cadre conceptuel suggéré par Mullender et Ward (1991), le modèle du groupe autogéré, est une intervention planifiée avec minutie, où les membres du groupe sont des partenaires de l'intervenant ou de l'intervenante dans toutes les facettes de l'intervention: élaboration des normes de fonctionnement, définition et analyse des problèmes, identification des objectifs. Les étapes préparatoires à I'action sont perçues comme cruciales, car elles permettent l'analyse sociale et la perspective écologique requises pour comprendre l'oppression vécue par les personnes participantes. À l'intérieur du groupe, les problèmes personnels peuvent se transposer en préoccupations collectives et le pouvoir peut se voir simultanément augmenté 
et partagé (ibid.: 28-29). Le passage à l'action est également déterminant, car chaque résultat aura tendance à renforcer l'estime de soi de chaque membre du groupe. Au bout du compte, l'évaluation continue de l'intervention se transforme en reformulation de l'analyse de départ et en identification des suites à donner. $C^{\prime}$ est ainsi que le groupe prend en charge l'intervention et atteint un stade d'empowerment (ibid.: 18-19).

Le groupe constitue donc un lieu privilégié pour l'empowerment et ce thème est fréquemment développé par d'autres auteurs (Hirayama et Hirayama, 1985; Pernell, 1985). L'avantage comparatif du groupe par rapport au traitement individuel repose sur le fait que l'activité collective permet d'élever plus facilement le niveau de conscientisation par rapport à l'oppression, par exemple à travers l'expérimentation du pouvoir à l'intérieur du processus d'aide. De plus, le groupe permet une relation de collaboration entre les membres, qui englobe la réciprocité et l'engagement mutuel entre chaque membre ainsi qu'un partage du pouvoir et de la lutte à mener (Lee, 1991: 12).

\section{L'organisation communautaire}

L'empowerment serait un thème autant implicite qu'explicite dans la littérature contemporaine sur l'organisation communautaire (Mizrahi et Morrison, 1993: 5). Contrairement aux méthodes précédentes, aucun cadre conceptuel précis n'est proposé car, de façon générale, l'empowerment est perçu comme faisant partie intrinsèque de cette méthode (Kahn, 1991:2 ; Mondros et Wilson, 1994: 4; Rothman, 1995 : 45). Les écrits recensés révèlent toutefois des nuances selon la stratégie d'organisation communautaire utilisée ${ }^{2}$ - action sociale, planning social, développement local de type communautaire.

Une étude aux États-Unis révèle que le but premier des organisations communautaires appartenant au modèle de I'action sociale (Mondros et Wilson, 1994) est I'accumulation et l'exercice du pouvoir ${ }^{3}$. Cet objectif peut s'avérer autant implicite qu'explicite, car, en cherchant le changement social, ces groupes visent simultanément le pouvoir (ibid.: 6 ; Rothman, 1995: 32). Deux variables influencent de façon déterminante les organisations d'action sociale. II s'agit du type d'intervention adopté - grassroots model, lobbying model, mobilizing model - et le champ dans lequel l'action est menée. D'une certaine façon, le choix de l'intervention découle d'abord du domaine, car c'est lui qui délimite le nombre potentiel de membres. Il s'agit d'un facteur clé, car le pouvoir d'une organisation d'action sociale repose en bonne partie sur le nombre de personnes qui l'appuient. Les modèles de lobbying et d'action politique (mobili- 
zing $\left.{ }^{4}\right)$ ne $s^{\prime}$ inscrivent toutefois pas dans une approche axée sur l'empowerment, car ni l'un ni l'autre ne perçoit le contrôle de I'intervention par les membres comme facteur significatif pour atteindre ses buts (233-244). Seul le modèle communautaire (grassroots), qui s'apparente à l'action communautaire de défense des droits sociaux (Mayer et Panet-Raymond, 1991), offre des possibilités sur ce plan malgré des contradictions importantes, car l'empowerment des membres y constitue l'objectif «interne» principal (Mondros et Wilson, 1994 : 227-251).

En planning social, le lien avec l'empowerment se fait à partir de la participation du milieu dans les décisions touchant l'organisation et la distribution des services. Une étude récente confirme que la participation des clients dans les décisions des organismes publics donne lieu à de meilleures interventions et à de meilleurs programmes (Itzhaky et York, 1994). Malheureusement, le nombre trop considérable de facteurs sous-jacents à cette participation n'a pas permis aux chercheurs de préciser davantage ce phénomène. Pour leur part, Hardina et Malott (1993) sont arrivées à la conclusion que l'empowerment des conseils d'administration des organismes parapublics de service sociaux requiert des membres qui, à la fois, ont le sentiment de maîtriser les problématiques et possèdent vraiment les compétences nécessaires pour prendre les décisions qui s'imposent. Cela fait en sorte que le milieu est souvent représenté par des gens qui font déjà partie de l'élite détenant le pouvoir et que les personnes représentant les usagers sont très limitées dans leur capacité d'influencer les décisions (ibid.: 7-10). Pour ces auteures, le discours étatique d'empowerment peut en réalité dissimuler un but de cooptation des populations démunies ou de transfert de responsabilités - un thème repris par Panet-Raymond et Bourque (1991) dans leur analyse de la relation asymétrique entre les organismes publics et parapublics et les organisations communautaires, relation qu'ils surnomment "pater-nariat». Hardina et Malott, tout comme Itzhaky et York, concluent que d'autres recherches sont nécessaires dans ce domaine. Tout compte fait, les effets d'empowerment semblent se limiter ici au partage d'informations et à l'ouverture des dispositifs de consultation pour recueillir l'opinion des clients (Rothman, 1995 : 42).

Bien que ce qui précède soit en accord avec la façon dont Rothman perçoit le planning social (structures formelles, activités techniques...), l'impression est donnée que ce modèle est limité aux institutions publiques et parapubliques - opinion mise en avant par Doucet et Favreau (1991 : 64), mais non par Rothman (1995: 30). De fait, il existe une parenté certaine entre le planning social et d'autres stratégies d'organisation de services, telle que le "function transfer» 
décrit par Grosser et Mondros (1985 : 169). Dans chaque cas, I'amélioration du bien-être repose sur les services techniques offerts par des structures détenant l'expertise. Ces services peuvent être exploités par des organismes de tout genre, incluant les organisations communautaires (ibid.: 165). II est donc possible que la fraction du mouvement populaire et communautaire québécois offrant des services sur une base autonome (Bélanger et Lévesque, 1992: 720 ; Doré, 1992: 143) emprunte elle aussi la voie du planning social. Est-ce que cela veut dire que ses effets d'empowerment seront aussi limités que ceux du secteur parapublic? La question clé est celle du contrôle des services et l'empowerment se joue sur ce plan. À titre d'exemple, les mécanismes de participation dans les cliniques populaires de santé (Bélanger et Lévesque, 1992: 721-722) rendent possible un contrôle réel qui ne se retrouve pas dans des établissements parapublics, les CLSC en particulier (Hardina et Malott, 1993: 7-10). En somme, la question d'empowerment est moins liée à la mise sur pied du service comme telle qu'au contrôle du milieu et à la possibilité pour les usagers et usagères des services $d^{\prime} y$ participer activement.

Le dernier modèle $d^{\prime}$ 'organisation communautaire, le développement local de type communautaire, s'insère directement dans le créneau de l'empowerment, car il contient tous les éléments essentiels relevés par Simon: participation de différentes couches de la population à la définition et à la résolution de leurs problèmes, rôle joué par le ou la praticienne de catalyseur, coordonnateur ou formateur selon le cas, conception du rôle du système client comme participant dans un processus d'interaction pour résoudre les problèmes (Doucet et Favreau, 1991: 30-31; Rothman et Tropman, 1987: 10)... Selon Miller (1991), I'empowerment doit être un but explicite pour chaque projet de développement local, à défaut de quoi on court le risque de pallier les problèmes au lieu de changer la situation. II faut aussi dépasser l'idée simpliste de l'autonomie complète de l'individu nouvellement apte à pêcher, car le proverbe chinois omet hélas! de dire qu'il ne peut s'appliquer que si de l'eau potable coule dans la rivière, que si des poissons $s^{\prime} y$ trouvent, que si l'on possède une canne à pêche, un hameçon ou deux et de l'appât. Bref, on ne peut pas fonctionner de façon autonome sans les ressources nécessaires. Selon Miller, le pêcheur empowered aura non seulement le contrôle de ces ressources, il sera même disposé à partager ce contrôle. Cette vision du développement axée sur l'empowerment est également mise en avant par d'autres auteurs (Abrahams, 1992; Friedmann, 1992) et elle lui confère, en bout de piste, un cachet politique fondé sur des principes de coopération et de solidarité, qui sont les bases d'une société plus juste et équitable. 


\section{L'administration des programmes}

En matière d'administration des programmes, certains auteurs préconisent l'empowerment comme fondement de l'évaluation des programmes. Fetterman (1994) en particulier propose un modèle $\mathrm{d}^{\prime}$ " empowerment evaluation" dont I'objectif principal est I'autoévaluation comprise comme le contrôle de l'évaluée sur l'ensemble du processus d'évaluation (sans toutefois en perdre la rigueur et le caractère scientifique). Pour lui, l'évaluation axée sur l'empowerment permet à l'individu de trouver de nouvelles possibilités d'action et de redéfinir son identité et son rôle à venir (ibid.: 9). Papineau et Kiely (1996a; 1996b) arrivent à des conclusions semblables sur le plan collectif à partir d'un modèle d'évaluation participative. Les effets d'empowerment produits par ce type d'évaluation se répercutent également sur le plan organisationnel par une plus grande efficacité des membres.

\section{La recherche}

En matière de recherche, la recherche participative comporte une priorité d'empowerment des groupes sans pouvoir. Whitmore (1988, 1991) en particulier soutient que cette activité dépasse la simple méthodologie de la recherche, car la recherche participative se caractérise, entre autres choses, par un contrôle commun de l'étude par les professionnels et les participants des méthodes qui misent sur la capacité des participants à produire les connaissances et par un processus qui démarre une action à long terme visant un changement social des personnes opprimées. L'insistance sur l'empowerment fait en sorte que la démarche cible davantage tout le processus de la production du savoir plutôt que des résultats précis. Dans une vision $d^{\prime}$ empowerment, la recherche participative aurait de nombreux effets positifs sur les individus et les collectivités - qui semblent s'apparenter à ceux du traitement individuel et du service social des groupes axés sur l'empowerment - dont, par exemple, une plus grande estime de soi, des habiletés nouvelles, une meilleure compréhension du système social et une amélioration du bien-être individuel et collectif (Whitmore, 1991: 54-56).

\section{LE PROCESSUS D'EMPOWERMENT}

Le pouvoir, selon Lemieux (1989: 181), "c'est de transformer un choix en une décision ». Il se situe donc dans un cadre d'action et son 
exercice repose sur une capacité de choisir, sur une capacité de décider en fonction du choix et, en fin de compte, sur une capacité d'agir en fonction de sa décision. Le développement de ces capacités constitue le processus d'empowerment. Ce processus est fort complexe, car il comporte des facettes multiples, variées et agencées aux réalités de la collectivité ou de l'individu ainsi que de son environnement. Une synthèse des ouvrages sur l'empowerment indique que chacune de ses dimensions renvoie à une progression logique s'échelonnant dans le temps sur au moins quatre plans:

\section{La participation}

Les études scientifiques (Schultz et al., 1995 ; Zimmerman et Rappaport, 1988) établissent hors de tout doute une corrélation entre la participation dans des organisations communautaires et l'empowerment "psychologique». Pour Breton (1994: 34), une telle participation renvoie à une progression où les gens passent: a) de l'assistance muette à la participation aux discussions simples (l'exercice du droit de parole); b) ensuite aux débats (I'exercice du droit d'être entendu); c) pour aboutir aux décisions (l'exercice du pouvoir ultime qui peut se manifester aussi bien de façon positive - donner son aval - que de façon négative - refuser son consentement).

\section{La compétence technique}

L'exercice du pouvoir repose sur trois capacités: choisir, décider et passer à l'action. En fait, pour plusieurs auteurs, I'empowerment doit se réaliser dans des actions concrètes (Breton, 1993: 25; Mullender et Ward, 1991: 18-19): "The power to name must be accompanied by the power to act» (Breton, 1994: 36). Presque tous les textes étudiés indiquent que I'acquisition progressive de connaissances pratiques et techniques est essentielle pour passer à l'action. De plus, les succès accumulés dans un champ précis renforcent la capacité d'agir dans d'autres sphères (Mondros et Wilson, 1995).

\section{L'estime de soi}

L'empowerment fait référence à la fois à une réalité subjective et à une réalité objective, où la subjectivité est la perception qu'on a de soi-même (Breton, 1994 : 28). En matière d'estime de soi, les évaluations négatives intériorisées et incorporées dans l'expérience de développement personnel constituent des blocs de pouvoir indirect 
qui doivent être surmontés (Lee, 1991; Lerner, 1986, dans Labonte, 1990 : 65). Se retrouve alors, dans le processus d'empowerment, une progression sur le plan psychologique qui débute par l'autoreconnaissance de la légitimité de son identité propre et ensuite de sa propre compétence, ce qui ouvre la porte à la reconnaissance de cette même compétence par les autres. Cela fait en sorte qu'une personne ou une collectivité ne peut pas passer au travers du processus pour quelqu'un d'autre (Simon, 1990: 32).

\section{La conscience critique}

La grande majorité des ouvrages étudiés font référence aux écrits de Paolo Freire et insistent sur le développement d'une conscience critique $^{5}$ comme composante essentielle à l'empowerment. Dans la pratique, celle-ci s'avère le produit d'une dynamique dialectique d'action et de réflexion (Staples, 1990: 38), surnommée "praxis » par Freire (1970), donc d'une interaction qui permet un cheminement s'échelonnant sur trois niveaux: 1) le développement d'une conscience collective (l'individu n'est pas seul à avoir un problème); le développement $d^{\prime}$ une conscience sociale (les problèmes individuels et collectifs sont influencés par la manière dont la société est organisée) ; 3) le développement d'une conscience politique (la solution de ces problèmes passe par une action de changement social, $c^{\prime}$ est-à-dire une action politique dans le sens non partisan du mot). Friedmann exprime bien la dimension séquentielle du processus lorsqu'il affirme que l'empowerment sur le plan politique requiert un processus préalable d'empowerment sur le plan social (1992:34). La progression s'oriente alors vers des changements structurels socioéconomiques ou politiques (Breton, 1993: 23) par le moyen d'une pédagogie qui repose sur le questionnement, voire la remise en question, et qui encourage les individus à trouver leurs propres réponses et solutions (Simon, 1990: 35).

\section{L'interaction des composantes}

Les composantes du processus d'empowerment s'entrelacent à l'image d'un câble confectionné de quatre cordes où chacune renforce les autres tout en se trouvant elle-même renforcée. Ce renforcement réciproque se réalise progressivement sur un continuum qui varie en fonction d'un nombre faramineux de variables. Bien que la façon dont chaque composante agit sur les autres ne soit pas claire, il est possible de postuler que c'est l'interaction des composantes qui caractérise l'empowerment, car chacune semble jouer un rôle précis 
TABLeAU 2

Composantes du processus général d'empowerment

\section{La participation}

assistance muette

$\rightarrow$ participation aux discussions simples (droit de parole)

$\rightarrow$ participation aux débats (droit d'être entendu)

$\rightarrow$ participation aux décisions (aval / refus de consentement)

\section{La compétence technique}

acquisition progressive de connaissances pratiques

et techniques requises par l'action

\section{L'estime de soi}

autoreconnaissance de la légitimité de l'identité propre

$\rightarrow$ autoreconnaissance de sa propre compétence

$\rightarrow$ reconnaissance de sa compétence par les autres

\section{La conscience critique}

conscience collective

(la personne / la collectivité n'est pas seule à avoir un problème)

$\rightarrow$ conscience sociale

(les problèmes individuels et collectifs sont influencés par la façon dont la

société est organisée)

$\rightarrow$ conscience politique

(la solution des problèmes d'ordre structurel passe par le changement social, $c^{\prime}$ est-à-dire l'action politique dans le sens non partisan du mot)

à l'intérieur du processus. Il en découle que l'absence d'une composante aurait pour effet de réduire sinon d'annuler la portée de l'empowerment à l'intérieur d'une intervention qui se voudrait axée sur ce processus.

Inévitablement, l'empowerment variera d'un individu ou d'une collectivité à I'autre, mais également d'événement en événement et d'organisation en organisation. En d'autres mots, une personne pourrait atteindre un assez haut degré d'empowerment à l'intérieur d'une organisation précise bien qu'en même temps, à l'intérieur d'un autre groupe où elle participe, la situation pourrait être carrément le contraire. En d'autres mots, l'empowerment ne serait pas nécessairement garanti dans un domaine malgré le fait que toutes les étapes du 
processus aient été franchies dans un autre. De plus, le processus $\mathrm{d}^{\prime}$ empowerment $\mathrm{n}$ 'est pas linéaire, mais plutôt multidimensionnel, et ses différentes dimensions sont en mutation continuelle, agissant de façon constante les unes sur les autres. Cela renvoie au caractère dialectique des phénomènes dynamiques et il n'y a donc pas d'état final d'empowerment (Staples, 1990:31).

Un regard sur certains liens entre différentes dimensions du processus ainsi que sur leur action réciproque peut aider à cerner certains enjeux supplémentaires pour le service social.

\section{Le lien entre la participation et la compétence technique}

Breton insiste sur le fait que les habiletés des uns ne doivent pas réduire le pouvoir des autres, et il ne faut donc pas isoler le développement d'habiletés individuelles des contextes interpersonnels, sociaux et structurels (1994:30-31). Une approche axée sur l'empowerment doit trouver des moyens pour encourager et soutenir la pleine participation de toutes les couches sociales du milieu par le développement de leurs compétences. C'est dans ce sens que les structures démocratiques sont encouragées sur le plan organisationnel (Schultz et al., 1995 : 312) pour favoriser l'empowerment, comme $c^{\prime}$ est le cas dans les pratiques d'organisation communautaire.

La participation dans les initiatives de développement local semble prometteuse à cet égard. Miller, par exemple, relève sept niveaux de participation: 1) l'individu bénéficie passivement des bienfaits résultant des efforts des autres; 2) il travaille au projet lancé par quelqu'un d'autre ; 3) il acquiert des habiletés techniques lui permettant $d^{\prime}$ entreprendre son propre projet; 4) il gère un projet ; 5) il exerce une autorité sur la gestion ; 6) il participe à l'élaboration des politiques et des programmes; 7) il est propriétaire, en tout ou en partie, des facteurs de production (1991: 5). Il est possible de déceler ici un cheminement qui passe de la défense d'intérêts purement individuels au contrôle des ressources collectives. L'approche axée sur l'empowerment doit stimuler et soutenir une telle progression, afin que toutes les personnes puissent à la fois exercer leurs droits et prendre leurs responsabilités en participant aux décisions qui les concernent, dont, par exemple, l'organisation et la distribution de services, I'orientation des nouveaux projets de développement, I'aménagement du territoire et I'utilisation des ressources locales. 


\section{Le lien entre la communication et l'estime de soi}

Selon une synthèse $d^{\prime}$ 'ouvrages sur le "community empowerment" (Parsons et al., 1994: 268-272), le cheminement sera vécu simultanément par la collectivité et par les individus qui en sont membres, et ce à travers quatre dimensions: la participation, la communication, la conscience critique et la compétence. Ces dimensions sont celles déjà présentées dans ce texte comme composantes du processus d'empowerment, sauf que l'estime de soi semble avoir été remplacée par la communication. Y a-t-il un lien à établir entre la communication et l'estime de soi?

Pour ces auteures, la relation entre un individu ou un groupe et la communauté requiert une certaine forme de communication. Cependant, quelle que soit sa forme, la communication sera basée sur I'interprétation personnelle des choses et des événements et il faut reconnaître que la perception de ce qui existe peut souvent se révéler fort différente de ce qui existe vraiment. L'expérience d'un individu ou d'un groupe peut teinter sa manière de voir et ceci renvoie aux blocages indirects de stigmatisation et de culpabilisation qui doivent être surmontés pour le développement de l'estime de soi (Lee, 1991 ; Lerner, 1986, dans Labonte, 1990 : 65). En matière de développement local de type communautaire, par exemple, l'objectif de la communication est d'établir un véritable partenariat au sein de la collectivité. Cela demande l'adoption d'une orientation pragmatique et un abandon d'attitudes dogmatiques sans toutefois exclure les vues divergentes ni les intérêts parfois opposés entre certains partenaires. Il faut néanmoins être en mesure d'exprimer son point de vue. En conséquence, lorsque l'estime de soi est faible, l'éventuel partenariat risque de l'être aussi. L'organisation communautaire peut s'avérer capitale à cet égard, car toute dynamique d'entraide dans un milieu aura tendance à diminuer les sentiments d'isolement et de culpabilité qui accompagnent l'absence de pouvoir (Breton, 1993: 7-11; Shragge, 1993 : iii; Lee, 1991).

\section{Le lien entre la conscience critique et la justice sociale}

La déficience de pouvoir peut s'avérer structurelle ou, en d'autres mots, des personnes peuvent subir une exclusion sociale ou économique tout simplement parce qu'elles font partie d'un groupe social spécifique (Russ, 1994 : 145-147). Même si certains membres d'un tel groupe peuvent individuellement s'approprier un degré de pouvoir et de compétence, en général la majorité des membres du même 
groupe demeurent opprimés (Staples, 1990: 32). C'est ainsi que l'action collective prend tout son sens, car elle vise le changement structurel par la modification d'un rapport de force collectif (Lindsay, 1992: 11; Pernell, 1985: 111). Même si tous les individus appartenant à un groupe s'avèrent gagnants lorsque les relations de pouvoir changent en leur faveur, le but explicite d'une telle intervention est l'amélioration de la situation spécifique pour le plus grand nombre de personnes possible et non pas seulement pour quelques fortunés (Staples, 1990: 34).

Plusieurs auteurs se démarquent donc du courant conservateur de self-empowerment (Ward et Mullender, 1991 ; Labonte, 1990) qui, de toute évidence, nie le recours à l'action collective ou au changement social pour améliorer le bien-être des collectivités démunies. Pour ces auteurs comme pour d'autres (Breton, 1993; Friedmann, 1992), un travail d'empowerment est indissociable de l'action pour enrayer l'oppression. Cela s'explique par le fait que seules les personnes et les collectivités sans pouvoir - disempowered, donc opprimées - ont besoin d'une intervention axée sur l'empowerment, car les personnes qui exercent déjà un contrôle sur leur vie n'ont pas vraiment besoin de ce genre d'approche.

Cette lutte contre l'oppression est sans contredit le thème qui revient le plus souvent dans les ouvrages sur l'empowerment. Cela vient certainement en partie de la nature intrinsèquement égalitariste de l'empowerment qui accorde d'office à chaque personne le droit de prise en charge individuelle de sa propre destinée (Staples, 1990:31). En réalité, l'empowerment ne vise pas l'exercice d'un pouvoir sans discrimination quant à son utilisation finale. Son but n'est pas le remplacement d'un pouvoir oppressif par un autre similaire. C'est ainsi que la notion de justice sociale - aussi nébuleuse soit-elle - revient régulièrement dans les écrits sur l'empowerment.

II ne faut toutefois pas sous-estimer les difficultés inhérentes à I' " exercice du pouvoir », surtout dans la sphère économique. De fait, l'empowerment peut même devenir un objectif dangereux, car on risque de leurrer les individus ou les communautés s'il n'y a aucune chance véritable qu'ils puissent atteindre leurs buts. II est donc plus probable que l'empowerment soit réalisable, quoique limité, dans une perspective réformiste que dans une voie révolutionnaire. Malgré ce bémol, une intervention communautaire axée sur l'empowerment a le potentiel d'apporter une contribution significative dans la lutte contre la pauvreté (Ninacs, 1996) et, à ce titre, mérite une évaluation plus approfondie. 


\section{Le lien entre la compétence et l'estime de soi}

L'ensemble du processus d'empowerment sera fondé sur la prémisse que les compétences requises pour effectuer le changement visé sont déjà présentes ou, au moins, que le potentiel pour les acquérir existe. Cela le situe dans le courant de "strengths perspective» (Alcorn et Morrison, 1994: 24; Saleebey, 1992: 8-13). Dans I'absence d'une reconnaissance de la compétence par autrui, selon Breton (1993: 4-5), la motivation personnelle sera mobilisée et les habiletés seront employées pour exercer une pression dans le but de faire changer l'opinion et d'être enfin reconnu pour ses compétences.

Cependant, le manque de compétence ou sa non-reconnaissance peut avoir un effet négatif. À titre d'exemple, dans l'évolution d'un groupe d'entraide économique en entreprise communautaire, l'émergence de leaders / entrepreneurs peut contribuer à un clivage entre les membres. Cet écart risque de s'accentuer au fur et à mesure que les opérations prennent de I'ampleur, car les personnes possédant moins d'habiletés peuvent se retrouver dépendantes des personnes mieux formées ou possédant autrement de meilleures ressources non financières (Laville, 1992: 92-93). Le processus d'empowerment pourrait alors se voir court-circuité pour certains membres du groupe. II y a peut-être un lien à établir sur ce plan avec les organisations communautaires d'action sociale où, en général, les intervenantes et intervenants rémunérés détiennent l'expertise et, pour ainsi dire, orientent I'action avec des leaders clés (Mondros et Wilson, 1994 : 227-251).

\section{Le lien entre l'empowerment individuel et I'empowerment collectif}

Bien que Staples ne désire pas opposer l'empowerment individuel et I'empowerment collectif (1990: 34), il affirme néanmoins que l'empowerment individuel serait un préalable pour le leadership du groupe quoiqu'il ne saurait garantir l'empowerment collectif (ibid. : 35). Labonte semble partager cette dernière affirmation. En fait, il voit l'ensemble du processus d'empowerment se déployer sur le continuum suivant: l'empowerment personnel, le développement de petits groupes, I'organisation communautaire, la revendication en coalition, I'action politique (Labonte, 1990:67-74). Cette progression semble déterminée, d'une certaine façon, par un nombre croissant de personnes dans le processus. Dans un même ordre d'idées, Friedmann lie les dimensions individuelle et collective en indiquant que l'empowerment des collectivités doit survenir tant sur le plan individuel que 
collectif et ce, en rapport avec chacune des trois formes de pouvoir requises pour assurer leur continuité et leur bien-être - pouvoir social, pouvoir politique et pouvoir psychologique (1992:32-33, 116).

Le processus collectif est influencé par un plus grand nombre de variables que le processus individuel, telles la qualité du leadership, les ressources, les opportunités et les stratégies adoptées... (Staples, 1990: 39). De plus, comme le souligne Rao (1992), l'hétérogénéité des groupes est une dimension souvent oubliée et son absence implique que les mesures instituées pour augmenter l'empowerment devront être adaptées aux forces et aux faiblesses de chaque souscatégorie de personnes. Ce bémol s'appliquerait d'office à l'intervention correspondante. Un certain paradoxe se révèle toutefois dans le fait que l'empowerment individuel peut, à certains moments, avoir un effet négatif sur l'empowerment collectif, par exemple lorsqu'un membre atteint un niveau de cheminement qui le conduit à l'extérieur du groupe qui a contribué à son empowerment. Cette situation se produit assez fréquemment dans plusieurs organisations communautaires où les individus retournent aux études, se trouvent un emploi, déménagent... Leur départ est très souvent un moment critique pour I'organisme, car ces personnes se situent souvent parmi les ressources humaines les plus engagées et les plus connaissantes.

L'empowerment collectif est-il synonyme de l'empowerment d'un milieu, d'une communauté? Certains semblent répondre oui lorsqu'ils affirment qu'une communauté empowered en est une où les gens, tant individuellement que par l'entremise de leurs organisations, mettent en commun leurs capacités et leurs ressources, ce qui mène, en bout de piste, à la compétence de la communauté (Schultz et al., $1995: 312)$. Or, une telle action présuppose une capacité d'action et, donc, le processus individuel préalable identifié par Staples (1990) et Labonte (1990). Ce point de vue semble assez répandu (Craig et Mayo, 1995; Hanna et Robinson, 1994). Cependant, même s'il n'existe à première vue aucune contradiction inhérente entre l'empowerment des individus et celui de la communauté, il faut distinguer les deux, puisqu'une communauté a une existence propre qui dépasse le simple cumul de ses membres. Des dimensions supplémentaires viennent ainsi s'ajouter aux composantes du processus $d^{\prime}$ empowerment déjà mentionnées dans ce texte. À titre $d^{\prime}$ exemple, l'empowerment communautaire renvoie: a) à l'intégration, dans des instances décisionnelles, $d$ 'individus non perçus comme leaders naturels (Biegel, 1984:130); b) au renforcement des réseaux naturels, communautaires et professionnels de soutien aux individus (ibid.: 
133-135); c) au moyen par lequel des groupes de personnes augmentent leur contrôle collectif (Fawcett et al., 1984: 146), et d) à l'équité dans la redistribution du pouvoir (Katz, 1984 : 204). Dans l'ensemble, l'empowerment de l'individu ne fait pas seulement partie intégrante de l'empowerment d'une collectivité, l'empowerment d'une collectivité doit même le favoriser (Lee, 1994: 263). De fait, ces deux processus sont réciproques et non pas en opposition (Staples, 1990:35).

\section{Les ressources requises}

Mondros et Wilson (1995) mettent en évidence trois ressources minimales pour la réalisation du processus d'empowerment: I'interaction, le temps et quelque chose qui a de la valeur.

L'interaction ici fait référence à la notion sociologique des actions et réactions découlant de processus interpersonnels en vertu non "...pas de la personnalité de l'individu, mais de ce qu'il est avec d'autres»(Grawitz, 1994 : 228). Cela renvoie au point de vue voulant que le groupe constitue un lieu privilégié pour une approche d'empowerment. En fait, Breton (1993: 2) croit qu'une approche axée sur l'empowerment ne peut se réaliser, dans le cadre du service social, que par le moyen d'un travail avec des groupes de personnes. Elle insiste, certes, sur un nombre de conditions qu'elle juge essentielles pour encadrer une pratique visant l'empowerment dans une perspective de service social des groupes dont, en particulier, l'identification de la justice sociale comme but poursuivi, l'orientation de la dynamique d'entraide dans ce sens et la reconnaissance du processus de conscientisation comme processus politique de libération (ibid.: 7-11).

Le processus d'empowerment ne se réalise pas du jour au lendemain, car il y a différents paliers à franchir. D'ailleurs, il faut prévoir beaucoup de temps (Kieffer, 1984:27-30), car on ne peut ni hâter ni forcer l'empowerment, on ne peut que le favoriser. Dans le cas d'une démarche collective, par exemple, la mise sur pied d'une coopérative de travail, les étapes et les obstacles sont nombreux et requièrent un soutien continu pendant l'ensemble du processus ainsi que beaucoup de temps. Le manque de temps peut, en effet, avoir des conséquences néfastes (Cosse, 1993: 172). En fait, le processus est souvent plus important que les résultats tangibles. Cela vient du fait que les efforts en tant que tels augmentent le sentiment d'appropriation chez les personnes qui les déploient (Shera, 1995: 1). De plus, le but $d^{\prime}$ un processus est également la réparation des « ruptures » 
antérieures (Tropman et Erlich, $1995: 227$ ), telles la stigmatisation et la culpabilisation des personnes qui ont subi une oppression.

Finalement, Mondros et Wilson (1995) croient que l'appropriation du pouvoir ne peut se faire sans quelque chose à retenir ou sur quoi on peut bâtir, ce qui renvoie à la notion d'investissement ou de risque. Un travail d'analyse de contenu a identifié le sens du risque comme élément capital du processus d'empowerment (Ninacs, 1994 : 22). La réussite d'une intervention serait-elle liée au fait que le processus procure aux gens quelque chose qu'ils peuvent perdre? II ne s'agit pas ici d'associer l'empowerment à un jeu de hasard, mais plutôt de voir le passage de la décision à l'action comme nécessitant une dimension de risque calculé - qu'on appelle "investir» dans le champ de l'économie et qui se trouve comme enjeu principal du développement. Une décision où on ne risque rien témoigne-t-elle vraiment de l'exercice d'un pouvoir? Et pas n'importe quel «investissement » : ne faut-il pas risquer son propre capital ? Dans une telle perspective, il nous semble qu'un travail social qui cherche à réduire les risques - comme c'est le cas plus souvent qu'autrement - peut entrer en contradiction avec une perspective $d^{\prime}$ empowerment.

\section{CONCLUSION}

La prolifération d'ouvrages sur l'empowerment depuis quelques années et l'enthousiasme des auteurs qui, il importe de le signaler, proviennent de toutes les sphères d'intervention du service social font voir que l'empowerment est en voie de devenir un phénomène incontournable dans le domaine du service social. Au moins une auteure propose l'empowerment comme concept unificateur du service social. Selon Parsons, et il semble que d'autres se rallient à cette opinion, au moins en partie (Johnson et Castengera, 1994), le service social reconnaît que la source des problèmes des individus est en interaction avec les structures et les dynamiques des institutions sociales et que les individus possèdent un rapport de pouvoir inégal face à ces institutions. Si le but du service social est de rendre les gens "capables d'établir eux-mêmes des interactions adéquates avec leur environnement, ou même d'être capables de produire eux-mêmes dans leur environnement les changements nécessaires» (Laforest, 1984: 28), les méthodes et stratégies de l'intervention sociale ne doivent-elles pas, en conséquence, être compatibles avec un objectif d'empowerment? 
En fait, l'empowerment correspond à un point de convergence des philosophies axées sur l'idée que les individus et les collectivités doivent avoir un mot à dire dans les décisions qui les concernent. $L^{\prime}$ exercice d'un pouvoir dans ce sens, c'est-à-dire celui qui permet d'augmenter le degré de contrôle sur soi-même et sur son environnement, et donc d'influencer son propre destin, est central dans un nombre croissant de théories et de pratiques dans des champs aussi divers que la psychologie communautaire, la santé publique et le développement économique, tant alternatif que communautaire. Cependant, malgré des assises scientifiques fort impressionnantes, le concept $d^{\prime}$ empowerment $n^{\prime}$ est pas universellement reconnu et il ne $s^{\prime}$ agit, certes, que d'une tendance parmi plusieurs autres (Simon, 1994 : 47). D'une certaine façon, on peut établir ici un parallèle avec l'approche communautaire qui également anime différentes pratiques sociales sans faire I'unanimité pour autant (Favreau et Hurtubise, 1993: 106-122; Gingras, 1991b). Selon Gingras, I'approche communautaire rallie de plus en plus d'adeptes en raison des courants actuels de la réduction des coûts sociaux et du renouvellement des pratiques sociales (1991a: 188-194). Selon Simon, I'intérêt grandissant pour l'empowerment serait fondé sur des préoccupations similaires (1994: xiii).

Dans une conjoncture socio-économique volatile où la seule constance semble être l'accroissement de l'appauvrissement et l'exclusion sociale, l'empowerment offre un certain espoir. Une approche d'empowerment voit une collectivité comme une chaîne. Puisqu'une chaîne est aussi forte que son maillon le plus faible, l'empowerment propose le développement d'un milieu par I'augmentation de la force de ses membres les plus fragiles. C'est donc une autre vision du développement proposée par l'empowerment, une vision qui semble très pertinente pour le service social. 


\section{Notes}

1. Dans ce texte, le mot "approche " est pris au sens figuré dans le sens d'une "démarche intellectuelle, [...] une attitude, comportant souplesse, prudence, et caractérisée par un état à la fois de grande vigilance et de grand respect pour l'événement ou l'objet "(Grawitz, 1993 : 303). Le mot " processus » renvoie au " mécanisme par lequel un phénomène se produit " (Lefrançois, 1991 : 137) et le mot " finalité " comme "but [...] sous la forme d'un processus dynamique, soumis non plus à une causalité linéaire, mais à une causalité circulaire de type probabiliste " (Grawitz, 1994 : 172-173).

2. La diffusion, depuis plusieurs années par Laval Doucet et Louis Favreau (1991), du courant explicatif de l'organisation communautaire élaboré par Jack Rothman en 1968 (Rothman, 1987) a permis qu'il soit aujourd'hui assez largement connu et c'est ce qui motive son utilisation ici, malgré qu'il soit incomplet sur plusieurs plans et que d'autres découpages auraient pu servir (Checkoway, 1995 ; Doré, 1985).

3. Ces auteurs font une nette distinction entre le pouvoir et l'empowerment. À leurs yeux, l'empowerment est un état psychologique, un sentiment de compétence et de contrôle (ibid. : 5), et ce serait le pouvoir comme tel qui s'avérerait processus et résultat.

4. Il ne faut pas confondre le mot mobilizing avec le modèle surnommé mobilizing model par les auteurs. L'expression " action politique " renvoie ici au type d'intervention communautaire et non pas à l'action de mobilisation.

5. La vraie traduction de " consciousness-raising " est " conscientisation ". Cependant, puisque ce mot renvoie à une " référence identitaire » en organisation communautaire (Doré, 1992 : 147), l'expression " conscience critique » est utilisée ici pour éviter toute confusion. 


\section{Références bibliographiques}

ABrahams, Caryl (1992). "A Social Development Practice Model for Community Development», Journal of the Community Development Society, vol. 23, $\mathrm{n}^{\circ} 2$ : 103-115.

ALCORN, Sandra et John D. MORRISON (1994). "Community Planning That Is "Caught" and "Taught" : Experiential Learning From Town Meetings", Journal of Community Practice, vol. 1, n $4: 27-43$.

BÉLANGER, Paul R. et Benoît LÉVESQUE (1992). "Le mouvement populaire et communautaire: de la revendication au partenariat (1963-1992)», dans Gérard Daigle et Guy Rocher (dir.), Le Québec en jeu : comprendre les grands défis. Montréal: Presses de l'Université de Montréal, p. 713-747.

BIEGEL, David E. (1984). "Help Seeking and Receiving in Urban Ethnic Neighborhoods : Strategies for Empowerment ", dans Julian Rappaport, Carolyn Swift et Robert Hess (dir.), Studies in Empowerment: Steps Toward Understanding and Action. New York: The Haworth Press, p. 119-143.

BRETON, Margot (1993). On the Meaning of Empowerment and Empowerment-Oriented Social Work Practice, communication, XVth Annual Symposium, Association for the Advancement of Social Work with Groups, New York, 21-24 octobre 1993, document ronéo, 15 pages.

BRETON, Margot (1994). "Relating Competence - Promotion and Empowerment », Journal of Progressive Human Services, vol. 5, $\mathrm{n}^{\circ} 1:$ 27-44.

CHECKOWAY, Barry (1995). "Six Strategies of Community Change », Community Development Journal, vol. 30, $\mathrm{n}^{\circ} 1: 2-20$.

COSSE, Pascaline (1993). "Vers le développement de l'autonomie économique des femmes : une expérience de groupe ", Service social, vol. 42, $\mathrm{n}^{\circ} 2: 163-182$.

CRAIG, Gary et Marjorie MAYO (sous la direction) (1995). Community Empowerment: A Reader in Participation and Development. New Jersey: ZED Books, 229 pages.

DAVIS, Elise (1989). The Concept of Empowerment in Social Practice. Toronto: Laidlaw Foundation, document ronéo, 24 pages.

DORÉ, Gérald (1985). "L'organisation communautaire: définition et paradigme », Service social, vol. 34, $n^{\text {os }} 2-3: 210-230$.

DORÉ, Gérald (1992). "L'organisation communautaire et les mutations dans les services sociaux au Québec, 1961-1991 : la marge et le mouvement comme lieu d'identité », Service social, vol. 41, n 2 : 131-162.

DOUCET, Laval et Louis FAVREAU (1991). "Mise en perspective autour de trois "modèles" " et "Stratégie et stratégies en organisation communautaire » dans Laval Doucet et Louis Favreau (sous la direction), Théorie et pratiques en organisation communautaire, Sainte-Foy: Presses de I'Université du Québec, p. 5-31 et 59-70.

FAVREAU, Louis et Yves HURTUBISE (1993). CLSC et communautés locales: la contribution de l'organisation communautaire. Sainte-Foy: Presses de I'Université du Québec, 211 pages. 
FAWCETT, Stephen B., Tom SEEKINS, Paula L. WHANG, Charles MUIU et Yolanda SUAREZ DE BALCAZAR (1984). "Creating and Using Social Technologies for Community Empowerment», dans Julian Rappaport, Carolyn Swift et Robert Hess (dir.), Studies in Empowerment: Steps Toward Understanding and Action. New York: The Haworth Press, p. 145-171.

FETTERMAN, David M. (1994). "Empowerment Evaluation», Evaluation Practice, vol. 15, no 1:1-15. Greenwich (CT) : American Evaluation Association/JAI Press, Inc., 15 pages.

FREIRE, Paulo (1970). Pedagogy of the Oppressed. New York: The Continuum Publishing Company, 186 pages.

FRIEDMANN, John (1992). Empowerment: The Politics of Alternative Development. Oxford, England et Cambridge, MA: Blackwell Publishers, 196 pages.

GINGRAS, Pauline (1991a). "L'approche communautaire», dans Laval Doucet et Louis Favreau (dir.), Théorie et pratiques en organisation communautaire. Sainte-Foy: Presses de I'Université du Québec, p. 187-200.

GINGRAS, Pauline (1991b). Le traitement en première ligne des demandes individuelles d'aide en CLSC selon une approche communautaire: guide de référence. Québec: ministère de la Santé et des Services sociaux en collaboration avec la Fédération des CLSC, 69 pages.

GraWITZ, Madeleine (1993). Méthodes des sciences sociales. Paris : Éditions Dalloz, 870 pages.

GRAWITZ, Madeleine (1994). Lexique des sciences sociales (6 édition). Paris: Éditions Dalloz, 399 pages.

GROSSER, Charles F. et Jacqueline MONDROS (1985). "Pluralism and Participation: The Political Action Approach», dans Samuel H. Taylor et Robert W. Roberts (dir.), Theory and Practice of Community Social Work. New York: Columbia University Press, p. 154-178.

HANNA, Mark G. et Buddy ROBINSON (1994). Strategies For Community Empowerment: Direct-Action and Transformative Approaches to Social Change Practice. New York: The Edwin Mellen Press, 223 pages.

HARDINA, Donna et Olga W. MALOTT (1993). Community Empowerment: Past Experience and Future Trends, communication au Sixième congrès biennal de la politique sociale organisé par le Community Services Council, Newfoundland and Labrador et le School of Social Work, Memorial University of Newfoundland, St. John's, Newfoundland, document ronéo, 26 pages.

HIRAYAMA, Hisashi et Kasumi HIRAYAMA (1985). "Empowerment through Group Participation: Process and Goal», dans M. Parnes (dir.), Innovations in Social Group Work: Feedback from Practice to Theory. New York: The Haworth Press, p. 119-131.

ITZHAKY, Haya et Alan S. YORK (1994). «Different Types of Client Participation and the Effects on Community Social Work Intervention », Journal of Social Service Research, vol. 19, n 1-2: 85-98. 
JOHNSON, Alice K. et Alice Rollins CASTENGERA (1994). «Integrated Program Development: A Model of Meeting the Complex Needs of Homeless Persons", Journal of Community Practice, vol. 1, $\mathrm{n}^{\circ} 3$ : 29-47.

KAHN, Si (1991). Organizing: A Guide for Grassroots Leaders. Silver Spring, Maryland: National Association of Social Workers, 343 pages.

KATZ, Richard (1984). "Empowerment and Synergy: Expanding the Community's Healing Resources", dans Julian Rappaport, Carolyn Swift et Robert Hess (dir.), Studies in Empowerment: Steps Toward Understanding and Action. New York: The Haworth Press, p. 201-226.

KIEFFER, Charles H. (1984). "Citizen Empowerment: A Developmental Perspective », dans Julian Rappaport, Carolyn Swift et Robert Hess (dir.), Studies in Empowerment: Steps Toward Understanding and Action. New York: The Haworth Press, p. 9-36.

LABONTE, Ronald (1990). «Empowerment : Notes on Professional and Community Dimensions ", Canadian Review of Social Policy / Revue canadienne de politique sociale, $\mathrm{n}^{\circ} 26: 64-75$.

LAFOREST, Jacques (1984). "La nature du service social (la compétence des travailleurs sociaux)", Intervention, mai, $n^{\circ} 68: 22-32$.

LAVILLE, Jean-Louis (1992). Les services de proximité en Europe: pour une économie solidaire, Paris, Syros/Alternatives, 247 pages.

LE BosSÉ, Yann D. et Marguerite LAVALLÉE (1993). «Empowerment et psychologie communautaire: aperçu historique et perspective d'avenir », Les Cahiers internationaux de psychologie sociale, 1993, $\mathrm{n}^{\circ} 18:$ 7-20.

LEE, Judith A.B. (1991). "Empowerment Through Mutual Aid Groups: A Practice Grounded Conceptual Framework », Groupwork, vol. 4, n 1 : 5-21.

LEE, Judith A.B. (1994). The Empowerment Approach to Social Work Practice. New York: Columbia University Press, 343 pages.

LEMIEUX, Vincent (1989). "Le pouvoir dans la réalisation des politiques sociales", Service social, vol. 38, $\mathrm{n}^{\text {os }} 2-3: 179-195$.

LINDSAY, Jocelyn (1992). Textes de base sur le modèle de groupe autogéré (volume). Sainte-Foy: Université Laval, École de service social, 48 pages.

MAYER, Robert et Jean PANET-RAYMOND (1991). "L'action communautaire de défense des droits sociaux», dans Laval Doucet et Louis Favreau (dir.), Théorie et pratiques en organisation communautaire. Sainte-Foy: Presses de I'Université du Québec, p. 97-118.

MILLER, G. David (1991). Why Teaching a Man to Fish is Not Enough: Empowerment as a Development Goal. Manchester, NH: New Hampshire College, 8 pages.

MIZRAHI, Terry et John D. MORRISON (1993). "Introduction » au volume sous leur direction, Community Organization and Social Administration: Advances, Trends and Emerging Principles. New York: The Haworth Press, p. 1-9.

MONDROS, Jacqueline B. et Scott M. WILSON (1995). Empowerment and Power in Social Work Practice, communication, 41st Annual Program Meeting, Council on Social Work Education, San Diego, 3-5 mars 1995, document ronéo, 3 pages. 
MONDROS, Jacqueline B. et Scott M. WILSON (1994). Organizing for Power and Empowerment. New York: Columbia University Press, 279 pages.

MOREAU, Maurice (en collaboration avec Lynne Leonard) (1989). Empowerment through a Structural Approach to Social Work: A Report from Practice. Ottawa: Carleton University, 326 pages.

MOreau, Maurice, Sandra Frosst, Gwyn Frayne, Mary HLYWA, Lynne LEONARD et Marilyn ROWELL (1993). Empowerment II: Snapshots of the Structural Approach in Acton. Ottawa: Carleton University, 360 pages.

MULLENDER, Audrey et David WARD (1991). Self-Directed Groupwork: Users Take Action for Empowerment. London, U.K.: Whiting \& Birch, 194 pages.

NINACS, William A. (1994). Empowerment and Community Economic Development, communication au Symposium organisé par la section Psychologie communautaire, Société canadienne de psychologie, à Penticton (Colombie-Britannique), document ronéo, 19 pages (résumé : Psychologie canadienne, $199435: 2 \mathrm{a}, 94)$.

NINACS, William A. (1995). "Entraide économique, création d'entreprises, politiques sociales et empowerment», Nouvelles pratiques sociales, vol. 8, n 1 : 97-119.

NINACS, William A. (1996). Le service social et la pauvreté : de la redistribution des ressources à leur contrôle? Sainte-Foy: Université Laval, École de service social, Laboratoire de recherche en service social [à paraître].

PANET-RAYMOND, Jean et Denis BOURQUE (1991). Partenariat ou pater-nariat. Rapport de recherche sur la collaboration entre établissements publics et organismes communautaires œuvrant auprès des personnes âgées à domicile, Groupe de recherche en développement communautaire, Université de Montréal. École de service social, 175 pages.

PAPINEAU, Danielle et Margaret C. KIELY (1996a). "Participatory Evaluation in a Community Organization: Fostering Stakeholder Empowerment and Utilization», Evaluation and Program Planning [à paraître].

PAPINEAU, Danielle et Margaret C. KIELY (1996b). "Peer Evaluation of an Organization Involved in Community Economic Development», Revue canadienne de santé mentale communautaire [à paraître].

PARSONS, Ruth, Jean F. EAST et Mary Beth BOESEN (1994). "Empowerment: A Case Study with AFDC Women", dans Lorraine Gutiérez et Paula Nurius (dir.), Education and Research for Empowerment Practice. Seattle, WA : Center for Policy and Practice Research, School of Social Work, University of Washington, p. 261-274.

PERNELL, Ruby B. (1985). "Empowerment and Social Group Work», dans M. Parnes (dir.), Innovations in Social Group Work: Feedback from Practice to Theory. New York: The Haworth Press, p. 107-117.

RAO, Mukunda (1992). Older Persons: Issues Concerning their Empowerment and Participation in Development. Communication, International Conference on Population Aging, University Center on Aging, San Diego State University, San Diego, Californie, 17-19 septembre, document ronéo, 15 pages. 
ROTHMAN, Jack (1995). "Introduction to Part One, Parameters of Intervention » et "Approaches to Community Intervention ", dans Jack Rothman, John L. Erlich et John E. Tropman (dir.), Strategies of Community Intervention. Itaska, IL: P.E. Peacock Publishers Inc., p. 3-63.

ROTHMAN, Jack et John E. TROPMAN (1987). "Models of Community Organization and Macro Practice Perspectives: Their Mixing and Phasing ", dans Fred Cox, John Erlich, Jack Rothman et John Tropman (dir.), Strategies of Community Organization. Itaska, IL : P.E. Peacock Publishers Inc., p. 3-26.

Russ, Jacqueline (1994). Les théories du pouvoir. Paris: Librairie Générale Française, 349 pages.

SALEEBEY, Dennis (1992). "The Philosophy of the The Strengths Perspective » dans Dennis Saleebey (dir.), The Strenghts Perspective in Social Work Practice. New York: Longman Publishing Group, p. 8-13 et 24-25.

SChulz, A.J., B.A. ISRAel, M.A. Zimmerman et B.N. CheCkOWAy (1995). "Empowerment as a multi-level construct: perceived control at the individual, organizational and community levels", Health Education Research: Theory and Practice, vol. 10, n 3: 309-327.

SHERA, Wes (1995). Organizational Empowerment. Communication, $41 \mathrm{st}$ Annual Program Meeting, Council on Social Work Education, San Diego, 3-5 mars 1995, document ronéo, 2 pages.

SHRAGGE, Eric (1993). "Preface» dans Eric Shragge (dir.), Community EConomic Development: In Search of Empowerment and Alternatives. Montréal: Black Rose, ix.

SIMON, Barbara Levy (1994). The Empowerment Tradition in American Social Work. New York: Columbia University Press, 227 pages.

STAPLES, Lee H. (1990). "Powerful Ideas about Empowerment», Administration in Social Work, vol. 14, $\mathrm{n}^{\circ} 2$ : 29-42.

TROPMAN, John E. et John L. ERLICH (1995). "Introduction to Part Two, Strategies " dans Jack Rothman, John L. Erlich et John E. Tropman (dir.), Strategies of Community Intervention. Itaska, IL: P.E. Peacock Publishers Inc., p. 223-240.

WARD, David et Audrey MULLENDER (1991). "Empowerment and Oppression: An Indissoluble Pairing for Contemporary Social Work », Critical Social Policy, n 32, automne, Essex, U.K., Longham Group, p. 21-30.

WEICK, Ann (1981). "Issues of Power in Social Work Practice», dans Ann Weick et Susan T. Vandiver (dir.), Women, Power, and Change. Washington, DC: NASW Press (National Association of Social Workers), p. 173-185.

WHITMORE, Elizabeth (1991). "Evaluation and Empowerment: It's the Process that Counts", Networking Bulletin: Empowerment and Family Support, Volume 2, Issue 2, Cornell University. Ithaca, New York: Cornell Empowerment Project, p. 1-7.

WHITMORE, Elizabeth et Patrick KeRANS (1988). "Participation, Empowerment and Welfare", Canadian Review of Social Policy / Revue canadienne de politique sociale, $\mathrm{n}^{\circ} 22: 51-60$.

ZIMMERMAN, Marc A. et Julian RAPPAPORT (1988). "Citizen Participation, Perceived Control and Psychological Empowerment », American Journal of Community Psychology, vol. 16, $\mathrm{n}^{\circ} 5$ : 725-750. 